\title{
Separability Criteria and Entanglement Measures for Pure States of $N$ Identical Fermions
}

\author{
A. R. Plastino \\ Instituto Carlos I de Física Teórica y Computacional, \\ Universidad de Granada, 18071-Granada, Spain, EU and \\ National University La Plata, UNLP-CREG, C.C. 727, 1900 La Plata, Argentina \\ D. Manzano and J. S. Dehesa \\ Instituto Carlos I de Física Teórica y Computacional, \\ Universidad de Granada, 18071-Granada, Spain, EU and \\ Departamento de Física Atómica, Molecular, y Nuclear, \\ Universidad de Granada, 18071-Granada, Spain, EU
}

(Dated: April 21, 2022)

\begin{abstract}
The study of the entanglement properties of systems of $N$ fermions has attracted considerable interest during the last few years. Various separability criteria for pure states of $N$ identical fermions have been recently discussed but, excepting the case of two-fermions systems, these criteria are difficult to implement and of limited value from the practical point of view. Here we advance simple necessary and sufficient separability criteria for pure states of $N$ identical fermions. We found that to be identified as separable a state has to comply with one single identity involving either the purity or the von Neumann entropy of the single-particle reduced density matrix. These criteria, based on the verification of only one identity, are drastically simpler than the criteria discussed in the recent literature. We also derive two inequalities verified respectively by the purity and the entropy of the single particle, reduced density matrix, that lead to natural entanglement measures for $N$-fermion pure states. Our present considerations are related to some classical results from the Hartree-Fock theory, which are here discussed from a different point of view in order to clarify some important points concerning the separability of fermionic pure states.
\end{abstract}

PACS numbers: 03.65.Ta, 03.65.Ud

Entanglement constitutes an essential ingredient of the quantum mechanical description of the physical world $[1,2]$. It is also a physical resource with important technological implications 3]. A fundamental first step in the study of the entanglement properties of a given class of quantum systems is the establishment of appropriate separability criteria. That is, to establish criteria that enables us to tell if a given quantum state is separable or entangled. A good separability criterion, besides its obvious importance as a tool for determining the presence or absence of entanglement, is also relevant as the possible basis of quantitative measures of entanglement. An appropriate measure of the deviation of the actual properties of a given quantum state from those required by the separability criterion may provide a valuable estimation of the amount of entanglement exhibited by that state.

The study of the entanglement features of systems consisting of $N$ identical fermions has attracted the attention of many researchers in recent years [4 13]. Entanglement between fermionic particles has been studied in connection with various physical scenarios. To mention just a few examples, researchers have recently investigated entanglement in two-electrons atomic states [14], entanglement between pairs of electrons in a conducting band [ 6$]$, entanglement dynamics in two-electrons scattering processes [7], and the role of entanglement in time-optimal evolutions of fermionic systems [12, 13], among many others. Appropriate separability criteria (and entanglement measures) for pure states of two identical fermions have been recently derived (using the Schmidt decomposition) and applied to the study of various physical systems and processes [6 [8]. Alas, the aforementioned derivations of separability criteria cannot be extended to situations involving more than two fermions because in such cases the Schmidt decomposition doesn't exist.

Some separability criteria for more than two fermions have been proposed in the recent quantum information literature, but they are difficult to implement in practice and exhibit a growing degree of complexity when one increases the number of particles of the system or the dimensionality of the single-particle Hilbert space. The necessary and sufficient criterion introduced by Eckert, Schliemann, Bruss, and Lewenstein [10] (from now on ESBL) is based on a projection operator acting upon an $N$-fermion state and resulting in an $(N-1)$-fermion state. This operator depends on an arbitrary singleparticle state $|a\rangle$. The ESBL criterion says that a pure $N$-fermion state $|\Psi\rangle$ has Slater rank one (that is, it is a separable state) if and only if the result of applying the projector operator on $|\Psi\rangle$ is, for any single-particle state $|a\rangle$, either equal to an $(N-1)$-fermion state of Slater rank 1 or equal to zero. The ESBL separability criterion has been recently hailed [4] as the main result known so far on necessary and sufficient separability criteria for $N$-fermion pure states. The ESBL criterion certainly is of considerable relevance from the fundamental and conceptual points of view, but it is of little practical use. 
To check if a given state $|\Psi\rangle$ fulfils the ESBL criterion is, in general, basically as difficult as the original problem of finding out if $|\Psi\rangle$ has Slater rank equal to 1 or not. The ESBL criterion can be iterated $N-2$ times, leading to a chain of separability tests eventually ending with a separability test to be performed on a two-fermion state. However, this procedure does not reduce the difficulty of the criterion, since each link in the aforementioned chain involves a relation that has to be checked for an arbitrary single particle state $|a\rangle$ [10]. A different approach employing sophisticated techniques from algebraic geometry has been advanced in [5]. According to this proposal, however, to be identified as separable a quantum $N$-fermion state has to comply with several relations (that is, not just with one identity as in the criterion proposed by us), their number increasing with the number of fermions in the system.

The aim of the present work is to derive two inequalities verified, respectively, by the purity $\operatorname{Tr}\left(\rho_{r}^{2}\right)$ and the von Neuman entropy $-\operatorname{Tr}\left(\rho_{r} \ln \rho_{r}\right)$ of the single particle reduced density matrix $\rho_{r}$ of an $N$-fermions pure state. These inequalities lead to simple separabilty criteria and suggest practical entanglement measures. These separability criteria turn out to be closely related to some previous results from the theory of Hartree-Fock wave functions that, even though themselves constituting useful necessary and suficient separability criteria, doesn't seem to have been recognized as such in the recent literature. Our derivations are different from (and simpler than) the ones followed in the aforementioned works on the Hartree-Fock wave functions. Moreover, our developments clarify why those previous results have not been believed to provide sufficient separability criteria for $N$ fermions states.

Let us consider a system consisting of a constant number $N$ of identical fermions with a single particle Hilbert space of dimension $D$, with $N \leq D$ (if $N>D$ it is not possible to construct an antisymmetric $N$-fermion state). A pure state of such a system is separable (that is, nonentangled) if it has Slater rank equal to one [4]. That is to say, the state is non entangled if it can be expressed as a single Slater determinant,

$$
a_{i_{1}}^{\dagger} \ldots a_{i_{N}}^{\dagger}|0\rangle
$$

where $a_{i}^{\dagger}$ are fermionic creation operators acting upon the vacuum state $|0\rangle$ and leading to an orthonormal basis $\left\{|i\rangle=a_{i}^{\dagger}|0\rangle\right\}$ of the single-particle Hilbert space. A pure state of the $N$-fermion system that cannot be written in the above way has a finite amount of entanglement. Correlations between the $N$ fermions that are due solely to guarantee the antisymmetric character of the fermionic states do not contribute to the state's amount of entanglement [8-10]. There are profound physical reasons for this. On the one hand, these correlations (exhibited by states with Slater rank 1) can't be used as a resource to implement non-classical information transmission or information processing tasks [10]. On the other hand, the non-entangled character of states represented by one Slater determinant is consistent with the possibility of associating complete sets of properties to both parts of the composite system (see [8, 9] for an interesting, detailed discussion of this approach).

When discussing the entanglement properties of systems of $N$ identical fermions the relevant group of "local transformations" is isomorphic to the group $S U(D)$ of (special) unitary transformations acting on the $D$-dimensional single-particle Hilbert space [10]. Given a transformation $U \in S U(D)$ the corresponding "local transformation" acts on a general $N$ fermions state according to $\sum w_{i_{1}, \ldots, i_{N}} a_{i_{1}}^{\dagger} \ldots a_{i_{N}}^{\dagger}|0\rangle \rightarrow$ $\sum w_{i_{1}, \ldots, i_{N}} \tilde{a}_{i_{1}}^{\dagger} \ldots \tilde{a}_{i_{N}}^{\dagger}|0\rangle$, where $\tilde{a}_{i}^{\dagger}|0\rangle=|\tilde{i}\rangle$ and $U|i\rangle=$ $|\tilde{i}\rangle,(i=1, \ldots, D)$. The set of non-entangled fermionic states is closed under the action of these "local transformations". Furthermore, the entanglement measures that we are going to consider in this work are invariant under those transformations.

A simple illustration of the fact that the correlations associated with a fermionic state of Slater rank 1 cannot be used as a resource for quantum information tasks is provided by a two-electrons system with a four dimensional relevant single-particle Hilbert space [10]. Let us assume that the relevant single-particle Hilbert space admits a basis of the for $\left\{\left|\phi_{1}\right\rangle|+\rangle,\left|\phi_{1}\right\rangle|-\rangle,\left|\phi_{2}\right\rangle|+\rangle,\left|\phi_{2}\right\rangle|-\rangle\right\}$, where $\left|\phi_{1,2}\right\rangle$ are two spatial wave functions and $| \pm\rangle$ corresponds to the spin degree of freedom. The two electrons can be treated as effectively distinguishable entities if they are spatially localized. This can occur if the moduli of $\left\langle\mathbf{r} \mid \phi_{1}\right\rangle$ and $\left\langle\mathbf{r} \mid \phi_{2}\right\rangle$ are non-overlapping. The single particle basis can then be partitioned between two agents (Alice and Bob), $\left\{\left|\phi_{1}\right\rangle|+\rangle,\left|\phi_{1}\right\rangle|-\rangle\right\}$ being the basis of Alice's space and $\left\{\left|\phi_{2}\right\rangle|+\rangle,\left|\phi_{2}\right\rangle|-\rangle\right\}$ the basis of Bob's space. Under these circumstances, a state of the form $\frac{1}{\sqrt{2}}\left(\left|\phi_{1}\right\rangle|+\rangle \otimes\left|\phi_{2}\right\rangle|-\rangle-\left|\phi_{2}\right\rangle|-\rangle \otimes\left|\phi_{1}\right\rangle|+\rangle\right)$ given by a single Slater determinant (and describing two particles localized in different spatial regions) effectively behaves as the non-entangled (in the usual sense) state $\left|\phi_{1}\right\rangle|+\rangle_{A} \otimes\left|\phi_{2}\right\rangle|-\rangle_{B}$ describing two distinguishable objects $(A$ and $B)$. On the other hand, a state describing two localized electrons that cannot be cast as one single Slater determinant effectively behaves as an entangled state (in the standard sense corresponding to distinguishable subsystems) that is useful for performing nonclassical information related tasks (see [10] for a more detailed discussion).

The amount of entanglement associated with an $N$ fermion state corresponds, basically, to the quantum correlations exhibited by the state on top of the minimum correlations needed to comply with the antisymmetric constraint on the fermionic wave function. Note that here we are considering entanglement between particles, and not entanglement between modes (see [15] for a comprehensive discussion of entanglement between modes).

Given a single particle orthonormal basis $\{|i\rangle=$ 
$\left.a_{i}^{\dagger}|0\rangle, i=1, \ldots, D\right\}$, any pure state of the $N$-fermion system can be expanded as,

$$
|\Psi\rangle=\sum_{i_{1}, \ldots, i_{N}=1}^{D} w_{i_{1}, \ldots, i_{N}} a_{i_{1}}^{\dagger} \ldots a_{i_{N}}^{\dagger}|0\rangle,
$$

where the complex coefficients $w_{i_{1}, \ldots, i_{N}}$ are antisymmetric in all indices and comply with the normalization condition

$$
\sum_{i_{1}, \ldots, i_{N}=1}^{D}\left|w_{i_{1}, \ldots, i_{N}}\right|^{2}=\frac{1}{N !} .
$$

The single-particle reduced density matrix $\rho_{r}$ associated with the $N$-fermion pure state (2) has matrix elements,

$$
\left\langle i\left|\rho_{r}\right| j\right\rangle=\frac{1}{N}\left\langle\Psi\left|a_{j}^{\dagger} a_{i}\right| \Psi\right\rangle,
$$

where the factor $1 / N$ guaranties that $\rho_{r}$ is normalized to unity,

$$
\operatorname{Tr} \rho_{r}=1 .
$$

Let $F_{i} \equiv\left\langle i\left|\rho_{r}\right| i\right\rangle$ denote the diagonal elements of $\rho_{r}$. After some algebra it is possible to verify that,

$$
F_{i}=\sum_{\substack{\left(i_{1}, \ldots, i_{n}\right) \\ i_{1}<i_{2}<\ldots<i_{n}}}(N !)^{2}\left|w_{i_{1}, \ldots, i_{N}}\right|^{2} f_{i}^{\left(i_{1}, \ldots, i_{N}\right)}, \quad i=1, \ldots, D
$$

where

$$
f_{i}^{\left(i_{1}, \ldots, i_{N}\right)}=\left\{\begin{array}{cc}
\frac{1}{N}, & \text { if } \\
0 & i \in\left(i_{1}, \ldots, i_{N}\right) \\
\text { otherwise }
\end{array}\right.
$$

Note that the sum in (6) has only $\left(\begin{array}{l}D \\ N\end{array}\right)=\frac{D !}{N !(D-N) !}$ terms because it doesn't run over all the $D^{N}$ possible $N$-uples $\left(i_{1}, \ldots, i_{N}\right)$; it runs only over the $\left(\begin{array}{l}D \\ N\end{array}\right) N$-uples whose indices are all different and listed in increasing order. Thus, the vector $\mathbf{F}$ (with components $\left\{F_{i}, i=1, \ldots, D\right\}$ ) can be expressed as a linear combination of the $\left(\begin{array}{l}D \\ N\end{array}\right)$ vectors $\mathbf{f}^{\left(\mathbf{i}_{1}, \ldots, \mathbf{i}_{\mathbf{N}}\right)}$ (with components $\left\{f_{i}^{\left(i_{1}, \ldots, i_{N}\right)}, i=1, \ldots, D\right\}$ ). Each one of these vectors has $D$ components, $N$ of them being equal to $1 / N$ and the rest equal to zero. To simplify notation it is convenient to introduce a single global label $k, 1 \leq k \leq\left(\begin{array}{l}D \\ N\end{array}\right)$, to characterize the coefficients $(N !)^{2}\left|w_{i_{1}, \ldots, i_{N}}\right|^{2}$ and the vectors $\mathbf{f}^{\left(\mathbf{i}_{1}, \ldots, \mathbf{i}_{\mathrm{N}}\right)}$. Equation (6) can then be recast in a more compact way as,

$$
F_{i}=\sum_{k=1}^{M} d_{k} f_{i k}
$$

where $M=\left(\begin{array}{l}D \\ N\end{array}\right)$ and the identifications

$$
\begin{aligned}
(N !)^{2}\left|w_{i_{1}, \ldots, i_{N}}\right|^{2} & \rightarrow d_{k} \\
f_{i}^{\left(i_{1}, \ldots, i_{N}\right)} & \rightarrow f_{i k}
\end{aligned}
$$

have been made. We have $0 \leq d_{k} \leq 1,(1 \leq k \leq M)$, $0 \leq f_{i k} \leq 1, \quad(1 \leq k \leq M ; 1 \leq i \leq D)$, and,

$$
\sum_{k=1}^{M} d_{k}=1 ; \quad \sum_{i=1}^{D} f_{i k}=1 ; \quad \sum_{i=1}^{D} f_{i k}^{2}=\frac{1}{N}
$$

The vector $\mathbf{F}$ and each of the vectors $\mathbf{f}_{\mathbf{k}}$ can be regarded as properly normalized probability distributions, and the vector $\mathbf{F}$ is a convex linear combination of the vectors $\mathbf{f}_{\mathbf{k}}$.

Let us now consider the sum of the squares of the components of the vector $\mathbf{F}$,

$$
\begin{aligned}
& \sum_{i=1}^{D} F_{i}^{2}= \sum_{i=1}^{D}\left\{\left(\sum_{k=1}^{M} d_{k}^{2} f_{i k}^{2}\right)+\right. \\
&\left.2\left(\sum_{k<k^{\prime}} d_{k} d_{k^{\prime}} f_{i k} f_{i k^{\prime}}\right)\right\} \\
&= \sum_{i=1}^{D}\left\{\left(\sum_{k=1}^{M} d_{k}\left(1-\sum_{k^{\prime} \neq k} d_{k^{\prime}}\right) f_{i k}^{2}\right)+\right. \\
&\left.2\left(\sum_{k<k^{\prime}} d_{k} d_{k^{\prime}} f_{i k} f_{i k^{\prime}}\right)\right\} \\
&= \sum_{i=1}^{D}\left\{\left(\sum_{k=1}^{M} d_{k} f_{i k}^{2}\right)-\left(\sum_{k \neq k^{\prime}} d_{k} d_{k^{\prime}} f_{i k}^{2}\right)+\right. \\
&= \sum_{i=1}^{D}\left\{\left(\sum_{k=1}^{M} d_{k} d_{k} d_{k^{\prime}}^{2} f_{i k} f_{i k^{\prime}}\right)\right\} \\
&\left.-\sum_{k<k^{\prime}} d_{k} d_{k^{\prime}}\left(f_{i k}^{2}+f_{i k^{\prime}}^{2}-2 f_{i k} f_{i k^{\prime}}\right)\right\} \\
&=\left\{\sum_{k=1}^{M} d_{k}\left(\sum_{i=1}^{D} f_{i k}^{2}\right)\right\} \\
&\left.\sum_{k<k^{\prime}} d_{k} d_{k^{\prime}} \sum_{i=1}^{D}\left(f_{i k}-f_{i k^{\prime}}\right)^{2}\right\}
\end{aligned}
$$

Since $\sum_{i=1}^{D} f_{i k}^{2}=\frac{1}{N}$ for all $k$, it follows from (11) that, 


$$
\begin{aligned}
\sum_{i=1}^{D}\left\langle i\left|\rho_{r}\right| i\right\rangle^{2} & =\sum_{i=1}^{D} F_{i}^{2} \\
& =\frac{1}{N}-\underbrace{\sum_{k<k^{\prime}} d_{k} d_{k^{\prime}} \sum_{i=1}^{D}\left(f_{i k}-f_{i k^{\prime}}\right)^{2}}_{\geq 0} \\
& \leq \frac{1}{N} .
\end{aligned}
$$

The inequality in (12) can also be obtained applying Jensen inequality to the square of the right hand side of (8) and taking into account the first and the third equations in (10).

The only way for the equality sign to hold in (12) is to have one of the $d_{k}$ equal to 1 and the rest equal to 0 , meaning that there is only one term in the original expansion for $|\Psi\rangle$. This implies that $|\Psi\rangle$ has Slater rank one, and can thus be expressed as one single Slater determinant. Since we didn't impose any restriction on the single-particle basis $\{|i\rangle\}$, equation (12) holds for any such a basis. In particular, it holds for the eigenbasis of the single-particle reduced statistical operator $\rho_{r}$, implying that

$$
\operatorname{Tr}\left(\rho_{r}^{2}\right) \leq \frac{1}{N}
$$

It is easy to see that when the Slater rank of the $N$ fermions state $|\Psi\rangle$ is one we have $\operatorname{Tr}\left(\rho_{r}^{2}\right)=\frac{1}{N}$. On the other hand, $\operatorname{Tr}\left(\rho_{r}^{2}\right)=\frac{1}{N}$ implies that there exists a single-particle basis for which the equal sign holds in (12), implying in turn that the state under consideration has Slater rank 1 and it is then separable.

Summing up, the following double implication obtains,

$$
|\Psi\rangle \text { has Slater rank one } \Longleftrightarrow \operatorname{Tr}\left(\rho_{r}^{2}\right)=\frac{1}{N} \text {. }
$$

In other words, a pure state of $N$ identical fermions is separable if and only if the purity of the reduced singleparticle density matrix is equal to $1 / N$.

It is possible to formulate a separability criterion equivalent to (14) in terms of the von Neumann entropy of the single particle density matrix $\rho_{r}$. Let us consider the Shannon entropies of $\mathbf{F}$ and $\mathbf{f}_{\mathbf{k}}$ (regarded as probability distributions),

$$
S[\mathbf{F}]=-\sum_{i=1}^{D} F_{i} \ln F_{i} ; \quad S\left[\mathbf{f}_{\mathbf{k}}\right]=-\sum_{i=1}^{D} f_{i k} \ln f_{i k}
$$

Using the concavity property of the Shannon entropy [16], it follows from (마) that,

$$
S[\mathbf{F}] \geq \sum_{k=1}^{M} d_{k} S\left[\mathbf{f}_{\mathbf{k}}\right]=\ln N
$$

where the inequality reduces to an equality if and only if all the probability vectors $\mathbf{f}_{\mathbf{k}}$ appearing in the sum in the middle term in (16) are equal to each other. This can only happen if one of the $d_{k}$ 's is equal to 1 and the rest are equal to zero. That is, it can happen only if the $N$-fermion state can be written as a single Slater determinant. Equation (16) holds for any single-particle basis $\{|i\rangle\}$. In particular, it holds for the eigenbasis of $\rho_{r}$, which leads to

$$
S\left[\rho_{r}\right] \geq \ln N
$$

It is plain that an $N$-fermion pure state with Slater rank one leads to a single-particle reduced density matrix verifying $S\left[\rho_{r}\right]=\ln N$. Conversely, the relation $S\left[\rho_{r}\right]=\ln N$ implies that there exists a single-particle basis such that $-\sum\left\langle i\left|\rho_{r}\right| i\right\rangle \ln \left\langle i\left|\rho_{r}\right| i\right\rangle=S[\mathbf{F}]=\ln N$ which, as we have already seen, implies that the $N$ fermion pure state can be written as a single Slater determinant and, consequently, describes a separable state. Summarizing,

$|\Psi\rangle$ has Slater rank one $\Longleftrightarrow-T r\left(\rho_{r} \ln \rho_{r}\right)=\ln N$.

A particular instance of the separability criterion (18), corresponding to systems of two identical fermions, has already been discussed by Ghirardi and Marinatto in [8]. The derivation of the $N=2$ case of (18) given by Ghirardi and Marinatto is based upon the Schmidt decomposition for systems of two fermions. Unfortunately, the Schmidt decomposition does not exist when $N \geq 3$ and, consequently, the developments presented in [8] cannot be extended to situations involving systems of three or more identical fermions. Our present treatment, besides providing a necessary and sufficient separability criterion valid for arbitrary values of the number $N$ of particles, is also of interest as yielding an alternative way of obtaining the $N=2$ criterion without recourse to the Schmidt decomposition.

The necessary and sufficient condition for separability $\operatorname{Tr}\left[\rho_{r}^{2}\right]=1 / N$ is closely related to the condition

$$
\rho_{r}^{2}=\frac{1}{N} \rho_{r}
$$

that the single particle reduced density matrix has to verify if the global wave function can be expressed as a Slater determinant. Condition (19) has been discussed in the past in the context of atomic physics [17, 18] and actually constitutes a classicall result from the theory of the Hartree-Fock approximation. However, the relevance of condition (19) as a useful separability criterion for $N$ fermions pure states has not been properly appreciated within the field of quantum entanglement theory. In fact, condition (19) has been in the recent literature regarded as not providing a necessary separability criterion. In fact, in connection with $N$-fermions states leading to a 
reduced density matrix verifying (19) it has been recently stated that "... a wave function of this kind can in general not be written as a single Slater determinant constructed from orthogonal states" [10]. As we are going to show next, our present results show in a direct and manifest way that the alluded wave functions can indeed be written as a single Slater determinant constructed from orthogonal states (that is, they have Slater rank 1).

Note that condition (19) is not, by itself, equivalent to either the relation (14) or to the entropic relation (18). It is plain that a density matrix $\rho_{r}$ complying with (19) must necessarily verify relations (14) and (18). However, the reciprocal implication doesn't hold. A density matrix verifying (14) (or verifying (18)) does not necessarily fulfil (19). For instance, if $\rho_{r}$ has eigenvalues $\left(\frac{1}{2}, \frac{1}{2 \sqrt{2}}, \frac{1}{2 \sqrt{2}}, 0\right)$ we have that $\operatorname{Tr}\left[\rho_{r}^{2}\right]=\frac{1}{2}$ but $\rho_{r}^{2} \neq \frac{1}{2} \rho_{r}$. However, it follows from our proof of the separability conditions (14) and (18) that either of the relations $\operatorname{Tr}\left[\rho_{r}^{2}\right]=\frac{1}{N}$ or $S\left[\rho_{r}\right]=\ln N$, together with the additional information that the single particle statistical operator $\rho_{r}$ comes from an $N$-fermion pure state, guarantee that equation (19) is verified (since in that case we have an equality in equation (12) and the global state must have Slater rank 1, implying that the only possible values for the eigenvalues of $\rho_{r}$ are $1 / N$ and 0$)$. In other words, in the special case of statistical operators $\rho_{r}$ that are reduced single particle matrices arising from an $N$-fermion state we have the double implication

$$
\operatorname{Tr}\left(\rho_{r}^{2}\right)=\frac{1}{N} \Longleftrightarrow \rho_{r}^{2}=\frac{1}{N} \rho_{r} .
$$

Consequently, and contrary to some current beliefs, equation (19) does provide a necessary and sufficient criterion for separability of $\mathrm{N}$-fermion states.

Finally, note that on the light of the separability criteria (14) and (18) it is reasonable to regard the differences

$$
\mathcal{E}_{L}=\frac{1}{N}-\operatorname{Tr}\left(\rho_{r}^{2}\right)
$$

$$
\mathcal{E}_{V N}=S\left[\rho_{r}\right]-\ln N,
$$

as measures of the amount of entanglement exhibited by a pure state of a system of $N$ identical fermions. The quantities (21) have already been proposed as measures of entanglement for fermions (particularly for two-fermion systems. See the excellent review [4] on entanglement in many-particle systems) but our present results lend considerable further support to that proposal, because we now know with certainty that the measures (21) are nonnegative quantities that vanish if and only if the fermionic pure state under consideration is separable. In the particular case of systems of two fermions with $D=4$ the quantity $4 \mathcal{E}_{L}$ reduces to the entanglement measure (usually referred to as squared concurrence) studied in [10] (see also [12]).

Summing up, we have derived a couple of inequalities involving respectively the purity and the von Neumann entropy of the single particle, reduced density matrix $\rho_{r}$ of an $N$-fermion pure state. These inequalities lead directly to simple and practical (necessary and sufficient) separability criteria based on the verification of one single identity. These criteria are drastically simpler than others that have been considered (for $N>2$ ) in the recent literature. Moreover, the aforementioned inequalities also suggest two practical measures of entanglement for fermionic pure states. In the particular case of $N=2$ the separability criteria discussed by us reduce to the criteria derived in [8] (see also [6]) by recourse to the fermionic Schmidt decomposition.

\section{Acknowledgments}

The authors gratefully acknowledge the Spanish MICINN grant FIS2008-02380 and the grants FQM-2445, FQM-481, and FQM-1735 of the Junta de Andalucía. They belong to the Andalusian research group FQM-207. D.M acknwoledges the corresponding FPI scholarship of the Spanish Ministerio de Ciencia e Innovación.
[1] Bengtsson I. and Zyczkowki K., Geometry of Quantum States: An Introduction to Quantum Entanglement (Cambridge University Press, Cambridge) 2006.

[2] Schlosshauer M., Decoherence and the Quantum-toClassical Transition (Springer, New York) 2007.

[3] Nielsen N. and Chuang I. L., Quantum Computation and Quantum Information (Cambridge University Press, Cambridge, 2000.

[4] Amico L., Fazio L., Osterloh A. and Vedral V., Rev. Mod. Phys., 80 (2008) 517.

[5] Lévay P. and Vrana P., Phys. Rev. A, 78 (2008) 022329.

[6] Naudts J. and Verhulst T., Phys. Rev. A, 75 (2007) 062104.

[7] Buscemi F., Bordone P. and Bertoni A., Phys. Rev. A, 75 (2007) 032301.
[8] Ghirardi G. and Marinatto L., Phys. Rev. A, 70 (2004) 012109.

[9] Ghirardi G., Marinatto L. and Weber T., J. Stat. Phys., 108 (2002) 49.

[10] Eckert K., Schliemann J., Bruss D. and Lewenstein M., Annals of Physics, 299 (2002) 88.

[11] Li Y. S., Zeng B., Liu X. S. and Long G. L., Phys. Rev. $A, 64$ (2001) 054302 .

[12] Borras A., Plastino A.R., Casas M. and Plastino A.R., Phys. Rev. A, bf 78 (2008) 052104.

[13] Oliveira V.C.G., Santos H.A.B., Torres L.A.M. and Souza A.M.C., Int. J. Quant. Inf., 6 (2008) 379.

[14] Amovilli C. and March N.H., Phys. Rev. A, 69 (2004) 054302.

[15] Banuls M-C., Cirac J. I., and Wolf M. M., Phys. Rev. A, 
76 (2007) 022311

[16] Cover T. M. and Thomas J. A., Elements of Information Theory (Wiley, New York) 1991.

[17] Rombouts S. and Heyde K., J. Phys. A: Math. Gen., 27
(1994) 3293.

[18] Lowdin P., Phys. Rev., 97 (1955) 1471. 